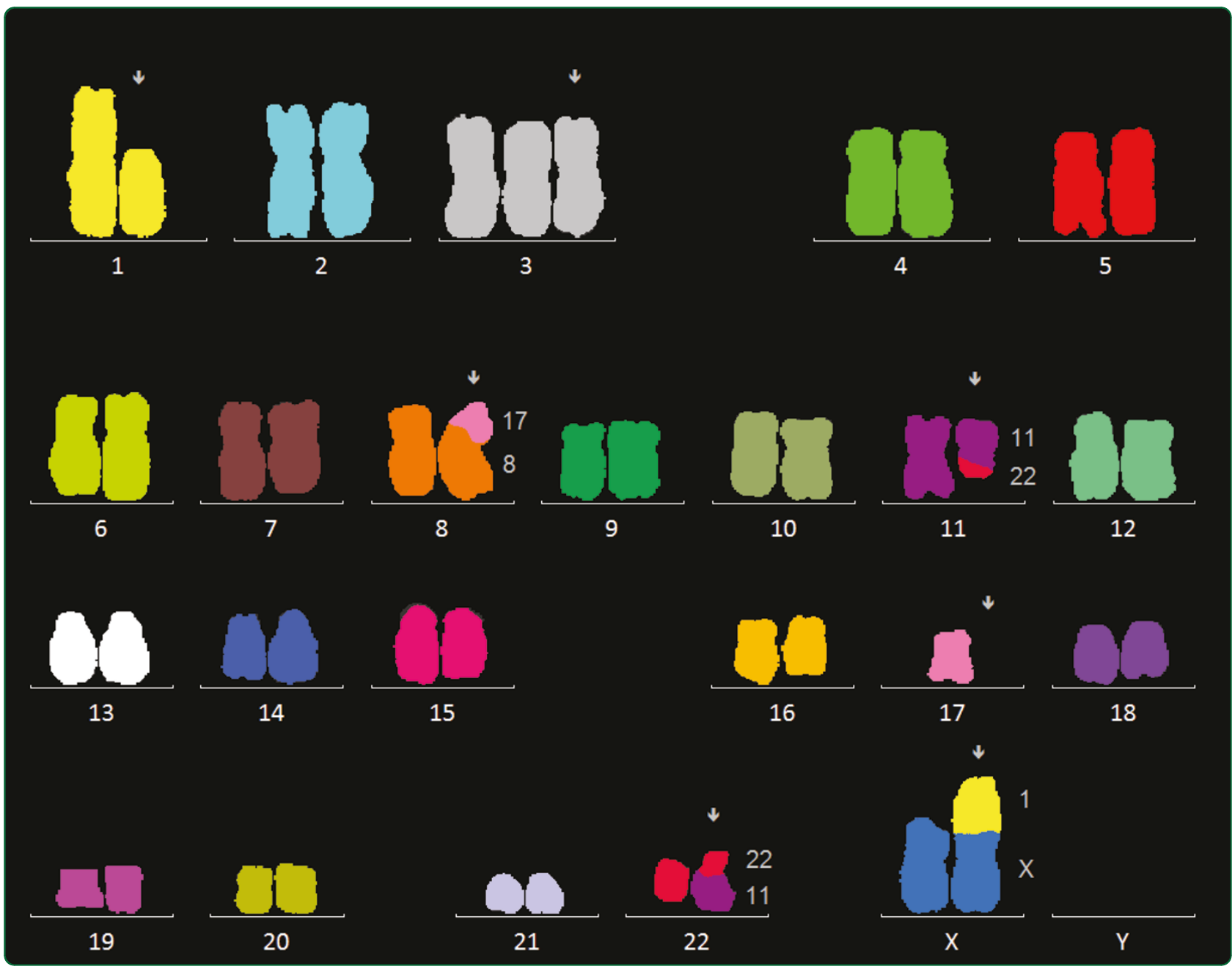

A rare case of $t(11 ; 22)$ in a mantle cell lymphoma like B-cell neoplasia resulting in a fusion of $I G L$ and CCND1: case report

Krings Rocha et al. 


\title{
A rare case of $t(11 ; 22)$ in a mantle cell lymphoma like B-cell neoplasia resulting in a fusion of IGL and CCND1: case report
}

\author{
Cristiano Krings Rocha, Inka Praulich, Iris Gehrke, Michael Hallek and Karl-Anton Kreuzer ${ }^{*}$
}

\begin{abstract}
The chromosomal translocation $(11 ; 14)(q 13 ; q 32)$ rearranging the locus for cyclin D1 (CCND1) to that of the immunoglobulin heavy chain $(I G H)$ can be found in virtually all cases of mantle cell lymphoma (MCL), while other CCND1 translocations are extremely rare. As CCND1 overexpression and activation is a hallmark of MCL it is regarded as a central biological mechanism in the development and maintenance of this disease. Here we present a patient initially diagnosed with chronic lymphocytic leukemia (CLL) where chromosome banding analysis revealed, among other aberrations, a translocation $(11 ; 22)(q 13 ; q 11.2)$. We show by fluorescence in situ hybridization (FISH) analysis that on chromosome 22 the immunoglobulin light chain lambda (IGL) is involved in this cytogenetic aberration. Additionally, we demonstrate the resulting overexpression of CCND1 on the RNA and protein level, thereby consolidating the new diagnosis of a MCL-like B-cell neoplasia. Summing up, we described a rare case of $\mathrm{t}(11 ; 22)(\mathrm{q} 13 ; \mathrm{q} 11.2)$ in a MCL-like neoplasia and showed that this aberration leads to an overexpression of CCND1 which is regarded as a key biological feature in MCL. This case underlines the importance of cytogenetic analyses especially in atypical cases of B cell lymphomas.
\end{abstract}

\section{Background}

Mantle cell lymphoma (MCL) and chronic lymphocytic leukaemia (CLL) belong to the group of CD5-positive small B-cell neoplasms. While CLL is the most common leukaemia in western countries with an incidence rate of about 5 cases per 100,000, MCL is much less frequent with a 10 -fold lower incidence rate of about 0.5 cases per 100,000 [1]. CLL and MCL share a distinct set of overlapping morphological features and clinical manifestation making it difficult in given situations to distinguish both diseases. Besides flow cytometric immunophenotyping, cytogenetic analysis is a deciding factor to discriminate between both entities. A prominent marker for MCL is the translocation $(11 ; 14)$ (q13; q32), resulting in a rearrangement of the gene loci for immunoglobulin heavy chain $(I G H)$ on chromosome 14 and cyclin D1 (CCND1) on chromosome 11. This leads to a constitutional overexpresion of CCND1 due to the IGH enhancer sequence located in front of CCND1 [2]. Cyclin D1 is a cell cycle regulator usually transiently

\footnotetext{
* Correspondence: karl-anton.kreuzer@uni-koeln.de

Department I of Internal Medicine, University at Cologne, Cologne, Germany
}

expressed in cells. Its aberrant overexpression in $\mathrm{MCL}$ leads to a high mitotic rate of the affected B-cells [3]. Other translocations resulting in an increased CCND1 gene expression are extremely rare [4-6].

In CLL cytogenetic studies focus on fluorescence insitu-hybridization (FISH) analyses for trisomy 12, deletion of $6 \mathrm{q}, 11 \mathrm{q}, 13 \mathrm{q}$ and $17 \mathrm{p}$, although about $30 \%$ of CLL cases show non-recurrent translocations [7]. The lack of mitotic CLL cells under culture conditions has largely been limiting the use of other chromosome analysis techniques. Recently, the use of new stimulating substances like CpG-oligonucleotide DSP-30 and Interleukin-2 resulted in an increase of proliferating CLL cells in culture and remarkably improved the success rate of classical chromosome analysis in CLL diagnostics [8-10]. Since then many cases of CLL where reported showing atypical and rare chromosomal aberrations, making the distinction borders to other B-cell malignancies blurry [11,12].

Despite these significant improvements, flow cytometric immunophenotyping remains the most important diagnostic tool for diagnosis of different B-cell neoplasms. While bright expression of FMC-7 and surface 
immunoglobulin (sIg) are usually seen in MCL but absent in CLL, the surface marker CD23 is typically found on CLL cells, but not MCL cells. Cases of CLL with variant phenotypes negative for CD23 and/or positive for FMC-7 usually are tested for $t(11 ; 14)(q 13 ; q 32)$ to eliminate the possibility of misdiagnosing MCL [13]. Since the clinical course of MCL is very aggressive with an overall survival of 3 to 5 years, all possible efforts for distinguishing CLL from MCL should be done.

\section{Case presentation}

In March 2010 we received peripheral blood (PB) from a 60 year old woman initially diagnosed with CLL in the context of a clinical CLL trial. The patient was therapeutically naive and exhibited an initial lymphocyte count of $22 \times 10^{6} / \mathrm{ml}$. By cell morphology a blastoid MCL variant could be excluded. Clinically, a general lymphadenopathy but no B symptoms were present. So far the disease developed slowly over 24 months suggesting an intermediate progressive course. The IgVH status was mutated. Unfortunately, no bone marrow or lymph node biopsy was available for further analysis.

\section{Materials and methods Flow cytometry}

Five-color flow cytometric analysis was performed on a FC500 instrument (Beckman Coulter, Brea/CA, USA) as described by Costa et al. [14]. The following antibodies were used: CD19-ECD, CD5-FITC, CD10-PE, CD23FITC, FMC7-FITC, CD79b-PE (Beckman Coulter, Brea/ CA, USA) and polyclonal rabbit anti-human lambda light chains, rabbit $\mathrm{F}(\mathrm{ab}$ ')2 $\mathrm{PE}$-conjugated (DAKO, Glostrup, Denmark).

\section{Chromosome analysis}

Peripheral blood was cultured for 96 hours in MarrowMax medium (Invitrogen, Grand Island/NY, USA). Two cultures with different mitogens were used: $12-O$-tetradecanoylphorbol-19-acetate (Sigma-Aldrich, St. Louis, USA) and CpG-oligonucleotide DSP30 (TIB MOLBIOL, Berlin, Germany) plus Interleukin 2 (Biochrom, Berlin, Germany). Chromosome analysis was performed on trypsin giemsa banded chromosome preparations and karyotypes were interpreted according to the ISCN 2009 [15].

\section{Fluorescence in-situ-hybridization (FISH)}

Interphase FISH was performed with a commercial set of probes consisting of 13q14/13q34 (D13S25, D13S319/ 13q34), 11q22/11cen (ATM/D11Z1), 6q23/6cen (MYB/ D6Z1), 17p13/17cen (TP53/D17Z1) and centromere 12 (D12Z3) (MetaSystems, Altlussheim, Germany). Additionally, dual-color, dual-fusion translocation probes for CCND1/IGH and for BCR/ABL were used (MetaSystems, Altlussheim, Germany). The gene rearrangement involving $I G L$ was assessed using an IGL dual-color, breakapart probe (MetaSystems, Altlussheim, Germany). The CCND1/IGL translocation was analysed with a tricolor, dual fusion probe consisting of a CCND1 dual color, break-apart probe (Abbott Molecular, Downers Grove, IL) and a homebrew IGL probe labeled in Spectrum Aqua (CTA-526G4, CTA-60B5, CTA-865E9). Interphase and metaphase FISH analysis was performed according to the manufacturer's instructions.

Multicolor FISH (mFISH) was performed on metaphase spreads using the 24XCyte MetaSystems Chromosome painting kit according to the manufacturer's instructions (MetaSystems, Altlussheim, Germany).

\section{Immunobloting}

Cells were lysed with MPER Mammalian Extraction Reagent (Thermo Scientific, Rockford/IL, USA) including DTT on ice for 60 minutes. Protein concentration was measured by Bradford assay. Solubilised proteins were resolved by PAGE and transferred onto a nitrocellulose membrane (Invitrogen, Karlsruhe, Germany). Blots were probed with monoclonal mouse anti-human cyclin D1 antibody (BD Biosciences, Franklin Lakes/NJ, USA), monoclonal mouse anti- $\beta$-actin antibody (SigmaAldrich, USA) and a polyclonal goat anti-mouse peroxidase-conjugated secondary antibody (Dako, Denmark). Antibody binding was detected using Amersham ECL ${ }^{\text {TM }}$ Western blotting detection reagents (GE Healthcare UK Limited, Buckinghamshire, UK). The well-characterized MCL cell line GRANTA-519, which features the $t$ $(11 ; 14)(\mathrm{q} 13 ; \mathrm{q} 32)$, hence shows a high expression of cyclin D1 protein [16] served as a positive control.

\section{Quantitative real-time RT-PCR}

For quantitative real-time RT-PCR, total RNA from the patient and GRANTA-519 cells was purified using QIAmp RNA Blood Mini Kit (Qiagen, Hilden, Germany) and cDNA synthesis was subsequently done applying First Strand cDNA Synthesis Kit (Roche, GrenzachWyhlen, Germany). The expression status of cyclin D1 (CCND1) was monitored using TaqMan॰ Probe realtime PCR assay with LightCycler@ FastStart DNA MasterPLUS HybProbe Kit (Roche, Grenzach-Wyhlen, Germany) on a LightCycler@ 480 Instrument. For amplification of CCND1, forward primer 5'-AGTGCAAGGCCTGAACCTG-3', reverse primer 5'-GGCAGTC TGGGTCACACTTGA-3' and the probe 5'-6FAMTTCCTGTCCTACTACCGCCTCACACGCTTC-Dabcyl-3' were applied with a standard PCR cycling profile of 45 cycles and an annealing temperature of $60^{\circ} \mathrm{C}$. Target gene expression was normalized against the expression of the housekeeping gene $A B L 1$ with primers and a probe as previously described [17] and target gene expression values are given as \%CCND1/ABL1 with 
PCR-efficiencies for CCND1 and ABL1 of 2,00 and 1,99, respectively.

\section{Results}

Flow cytometric analysis showed a positive population for CD5, FMC-7, CD79b and surface Ig lambda but negativity for CD10 and low CD23 (Figure 1). This immunophenotype was more consistent with a MCL than with CLL. Morphology on peripheral blood smears showed no smudge or basket cells which are typical for CLL patients. Instead lymphoid cells with irregular nuclear contours were seen, fitting the antecedent suspicion of MCL (Figure 2). For further confirmation of the supposed MCL a FISH for $\mathrm{t}(11 ; 14)(\mathrm{q} 13 ; \mathrm{q} 32)$ was performed. Surprisingly, the rearrangement of $I G H$ and CCND1 could not be confirmed, but an additional signal for $11 \mathrm{q} 13$ was observed in $78 \%$ of analysed cells (Figure 3a). Two constellations were possible: Trisomy 11 or translocation of chromosome 11 involving the CCND1 gene. Chromosome analysis showed two different clones, both with a trisomy 3 and a translocation $\mathrm{t}(11 ; 22)(\mathrm{q} 13$; q11.2), proving the second constellation to be true. Karyotype was confirmed via mFISH and described as 47 , $\mathrm{XX},+3, \mathrm{t}(11 ; 22)(\mathrm{q} 13 ; \mathrm{q} 11.2)[16] / 46, \mathrm{X}, \operatorname{der}(\mathrm{X}) \mathrm{t}(\mathrm{X} ; 1)$ (p22.1;p21), del(1)(p21), +3, der(8)t(8;17)(p21;q21), $\mathrm{t}(11 ; 22)(\mathrm{q} 13 ; \mathrm{q} 11.2),-17,2 \sim 9 \mathrm{dmin}$ [4] (Figure 4 and 5).
Further analysis applying our routinely used CLL FISH-panel showed a normal signal pattern for 6q23/ 6cen, 13q14/13q34, and centromere 12. Interphase FISH for $11 \mathrm{q} 22 / 11$ cen had a normal signal pattern, whereas on metaphases the translocation $\mathrm{t}(11 ; 22)$ could be confirmed, given that signal for $11 \mathrm{q} 22$ and for 11 centromere were localized on different chromosomes (data not shown). Interphase and metaphase FISH for $17 \mathrm{p} 13 /$ 17 cen were aberrant on cells with the complex clone showing one signal for $17 \mathrm{p} 13$ (aberrant in $10 \%$ of analysed interphases), well-fitting the unbalanced translocation $\mathrm{t}(8 ; 17)$ with loss of $17 \mathrm{p}$ (data not shown).

To determine the translocation partner gene for $C C N D 1$ on chromosome 22 three different FISH probes were used: one dual-color, dual-fusion probe for $B C R /$ $A B L$, a dual-color, breakapart $I G L$ probe and a tri-color, dual fusion CCND1/IGL probe. The $B C R / A B L$ probe showed a normal signal pattern (data not shown) whereas the IGL probe showed a break in one IGL gene locus in $84 \%$ of analysed cells (Figure 3b). FISH analysis with the CCND1/IGL tri-color, dual fusion probe could clearly show the CCND1/IGL translocation on $78 \%$ of analysed interphases and also on metaphases (Figure 3c).

As investigated by quantitative real-time RT-PCR, an overexpression of $C C N D 1$ could be clearly detected for the patient $(\% C C N D 1 / A B L 1=144,45)$ compared to

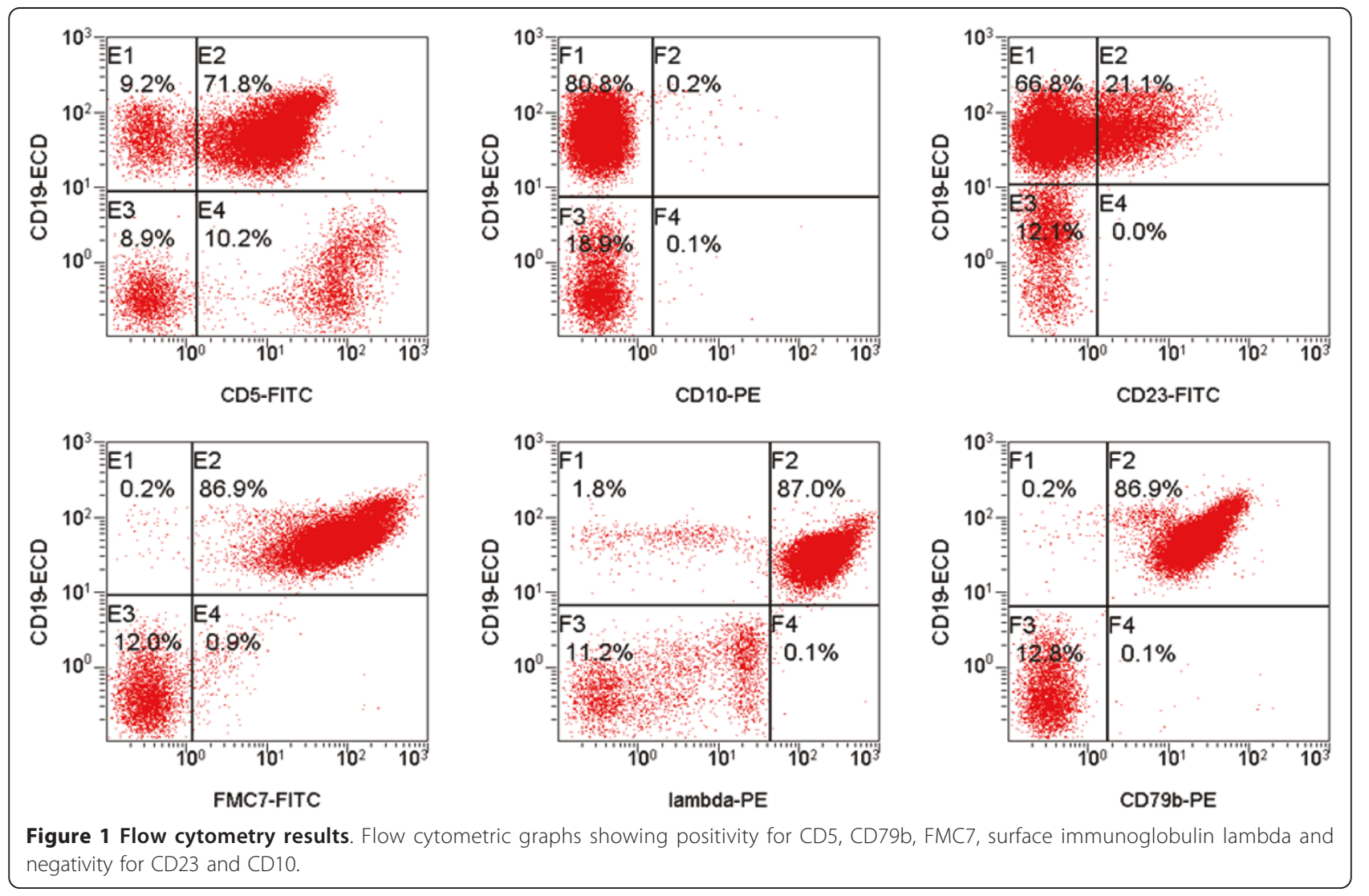




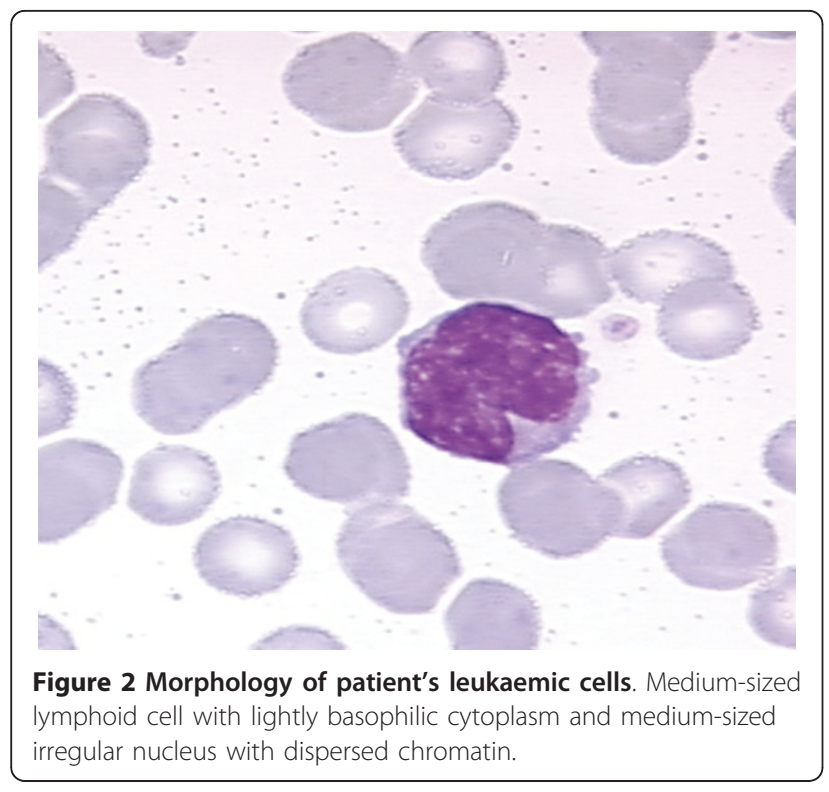

GRANTA-519 cells which served as positive control $(\% C C N D 1 / A B L 1=146,85)$. Only a minimal expression of CCND1 (\%CCND1/ABL1 = 0,37) could be detected for the negative control (Figure 6). In conformity with those findings, western blot analysis of cyclin D1 protein showed a remarkably overexpression (Figure 7).

\section{Discussion}

In general the vast majority of MCL patients show a translocation $(11 ; 14)$ (q13;q32) leading to an $I G H / C C N D 1$ gene rearrangement. Atypical translocations involving CCND1 and immunoglobulin kappa chain (Igk) also leading to an overexpression of cyclin D1 have been reported
[18]. IGL translocations were found in different neoplasms, e.g. $\mathrm{t}(18 ; 22)$ leading to $B C L 2$ to $I G L$ rearrangement in a case of CLL [19] or $c-M Y C$ to IGL rearrangement in Burkitt's lymphoma [20]. Komatsu et al. [4] reported on a MCL case showing an atypical rearrangement $t(11 ; 22)$ (q13;q11). They first speculated this translocation might result in a rearrangement of CCND1 to the IGL gene locus and confirmed their hypothesis on molecular level in a subsequent publication [21]. The WHO classification points to the possibility of this rare translocation, referring to aforementioned work [22].

In our case we investigated the affected gene on chromosome 22 involved in the $t(11 ; 22)$ and therefore responsible for the cyclin D1 overexpression, possibly leading to the development of an MCL. A FISH analysis with a $B C R /$ $A B L$ probe was performed, due to the localization of $B C R$ on 22q11 and different reports showing atypical cases of $B C R$ translocations to e.g. FGFR1 or PDGFRA genes $[23,24]$. As we found a normal signal pattern for $B C R / A B L$ and therefore excluded the involvement of $B C R$ to the translocation on chromosome 22 we assumed an involvement of IGL. FISH analysis as the method of choice for proving IGL involvement has already been reported for different chromosome 22 translocations [25]. Thus, our FISH analysis with a CCND1/IGL probe could prove the fusion of CCND1 to IGL concordantly to the findings by Komatsu et al $[4,21]$. Furthermore, we could show the cyclin D1 overexpression on mRNA and protein level, consolidating the diagnosis of a MCL-like neoplasia.

Analysis on GTG banded chromosome preparations revealed two different aberrant clones. The dominant clone, presumably the mainline, revealed an additional chromosome 3 and the translocation between

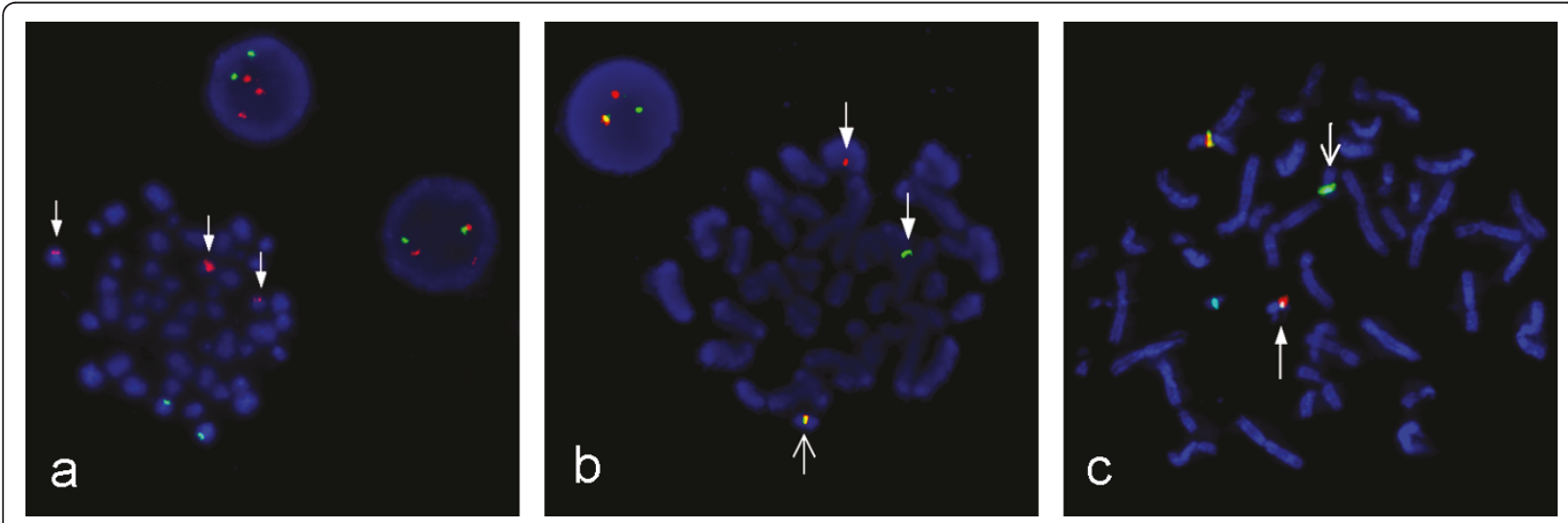

Figure 3 Metaphase and interphase FISH. (a) FISH showing 3 signals for CCND1 due to a translocation at the CCND1 locus on 11(q13). CCND1 on 11 (q13) is marked in red, IGH on 14(q32) is marked in green. (b) FISH confirming IGL rearrangement with an IGL dual-color breakapart probe. Broad arrows show separated signals for the IGL proximal region in red and for the distal region in green. The open arrow shows a fusion signal for the normal chromosome 22. (c) FISH with a CCND1/IGL tri-color, dual-fusion probe, showing the IGL/CCND1 translocation. CCND1 on 11(q13) marked in Spectrum Orange and Spectrum Green, IGL on 22(q11.2) marked in Spectrum Aqua. The broad arrow shows the derivative chromosome 22 and the open arrow the derivative chromosome 11. 


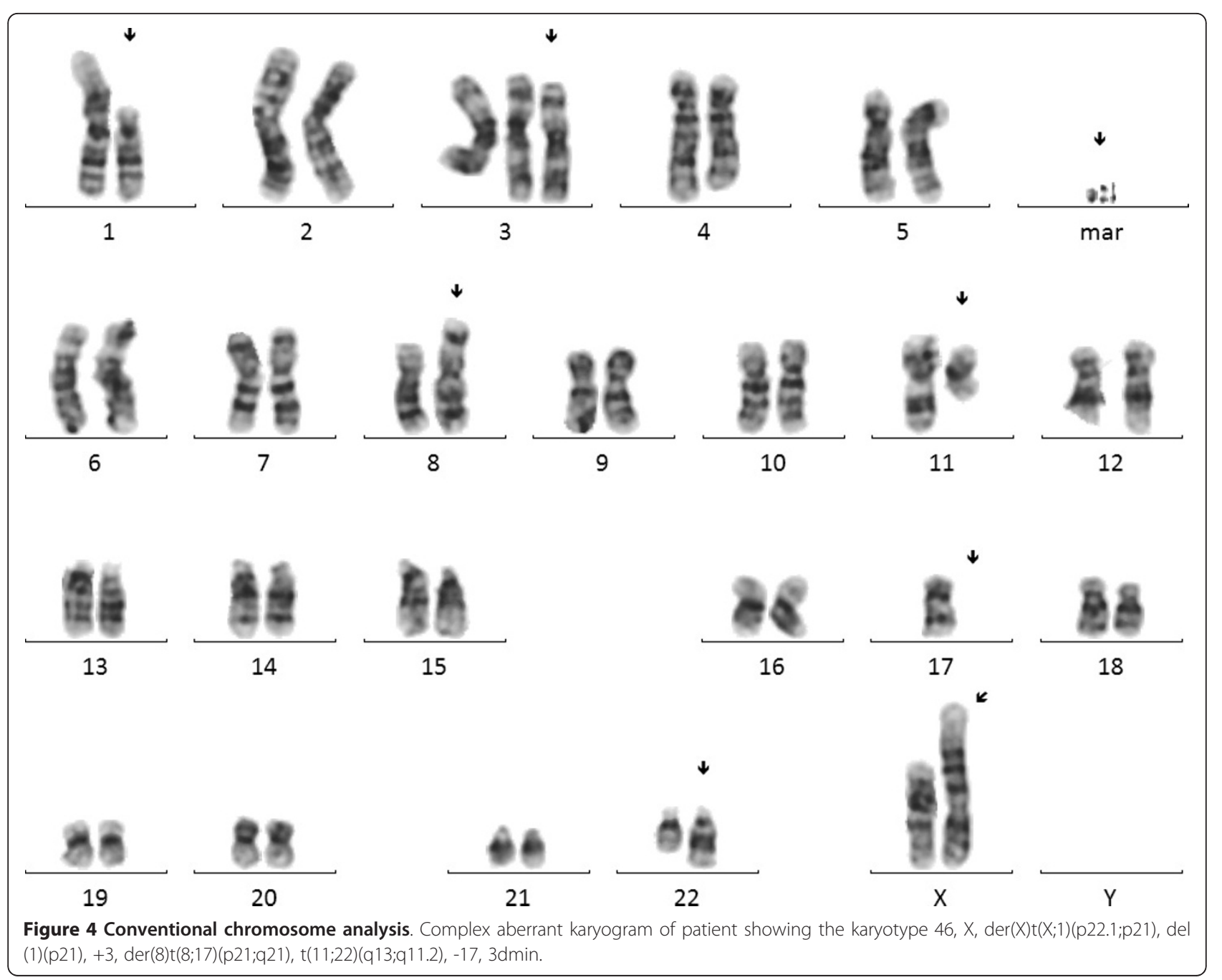

chromosomes 11 and 22. The second clone showed different additional secondary abnormalities affecting chromosomes X, 1, 8 and 17. As reported before, CCND1 translocations normally are considered as the primary cytogenetic event in MCL. Secondary events are seen in up to $88 \%$ of MCL cases, often involving trisomy 3, breaks in 1p21 and 17p deletions among others [26], in conformity with our findings in this case. The discovery of double minutes (DM) was quite surprising, as double minutes are very rare in lymphatic neoplasms. DM are acentric extrachromosomal chromatin which may lead to an amplification of oncogenes, like $c-M Y C$ amplification in colorectal carcinoma [27]. Unfortunately, there was no investigation material left for further analyzing the DM, e.g. by array-CGH to finally clarify the source of this additional genetic material.

\section{Conclusions}

Our patient was first suspected to have a CLL. We could show, that the $t(11 ; 22)(q 13 ; q 11.2)$ leads to an overexpression of cyclin D1 due to the rearrangement of $C C N D 1$ to $I G L$. In addition immunophenotype and morphology of the cells showed a typical MCL configuration. However, some cases of $t(11 ; 14)$ positive CLL [9] and on the other hand $t(11 ; 14)$ negative MCL have been reported [28]. Cyclin D1 is usually overexpressed in MCL, but also overexpression of cyclin D2 or cyclin D3 may induce a MCL [5]. In general, B-cell neoplasms carrying rare translocations involving CCND1 and Ig kappa or Ig lambda, like reported here, are diagnosed as MCL. A distinction between indolent and common cases of MCL has been proposed lately by Fernandez et al. [29]. On the other hand cyclin D1 overexpression in three cases with an IGKCCND1 rearrangement have been diagnosed as small-cell B-non-Hodgkin lymphoma, as they did not show typical features of MCL [18]. In our case we could show some typical features of a MCL like a CCND1 translocation, recurrent secondary chromosomal aberrations and cyclinD1 overexpression. But also some atypical features were found like the existence of double minutes and mutated 

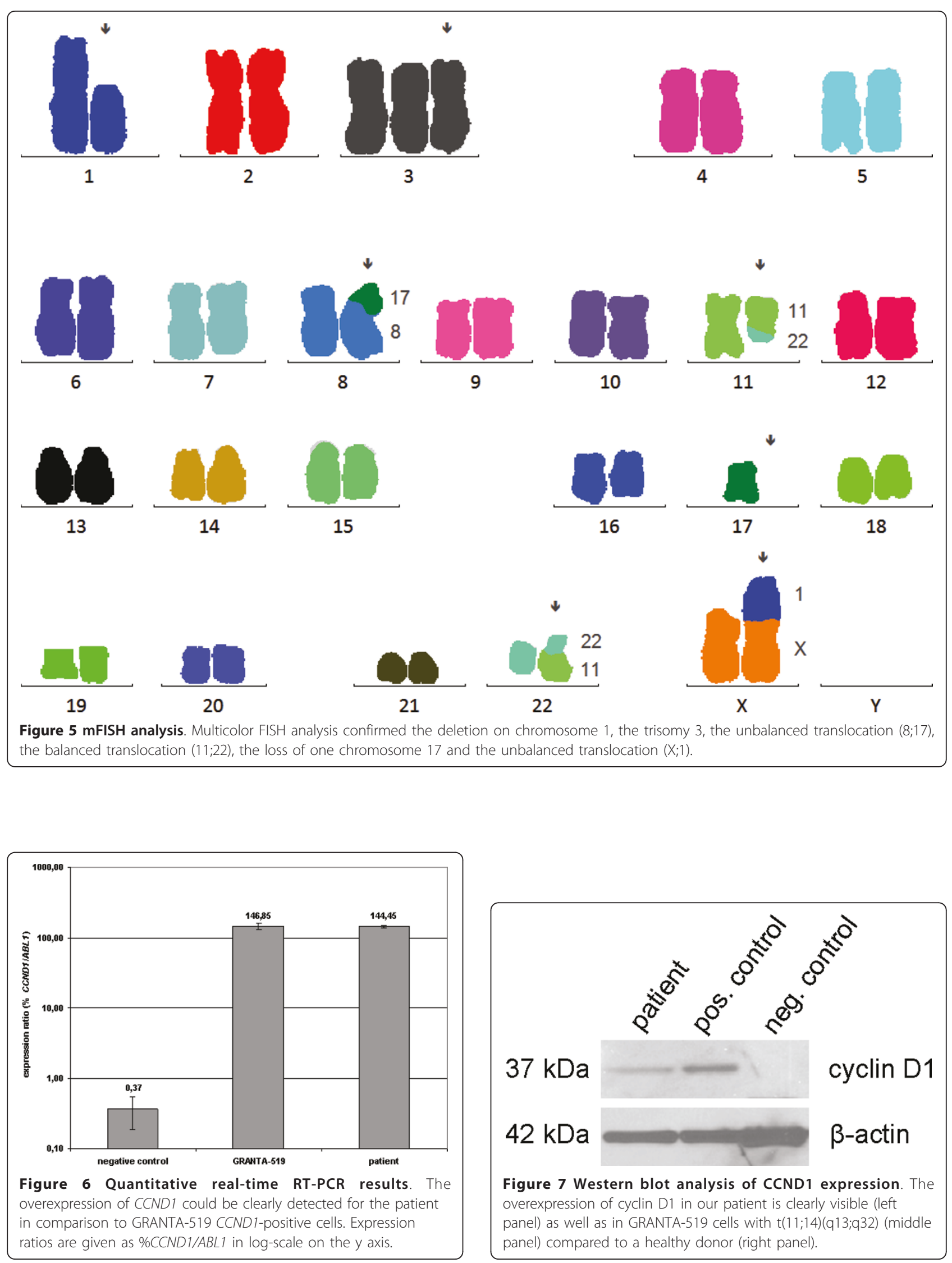
$\operatorname{IgVH}$ status, and therefore we suggest calling this a case of a MCL-like B-cell neoplasia.

Summing up, we described a third case of a $\mathrm{t}(11 ; 22)$ (q13; q11.2) leading to a rearrangement of CCND1 to $I G L$ and showed that this aberration leads to an overexpression of CCND1 which is regarded as a key biological feature of MCL. This case underlines the importance of cytogenetic analyses especially in atypical cases of B cell lymphomas.

\section{Consent}

Written informed consent was obtained from the patient for publication of this case report and any accompanying images. A copy of the written consent is available for review by the Editor-in-Chief of this journal.

\section{Acknowledgements}

We would like to thank Stephan Gesk for helping us with the CCND1/IGL FISH probe. We would also like to thank Stephanie Hippler, Anja Reuter, Sabine Hallmann, Eva Vogt, Sabrina Aschermann and Barbara Pentok for their excellent technical assistance.

\section{Authors' contributions}

CKR performed the cytogenetic and FISH analysis. IP performed the PCR analysis, IG performed the Protein analysis. MH and KAK supervised the experiments and helped in drafting the manuscript. CKR drafted the manuscript and all authors read and approved the final manuscript.

\section{Competing interests}

The authors declare that they have no competing interests.

Received: 10 December 2010 Accepted: 1 April 2011

Published: 1 April 2011

\section{References}

1. Morton LM, Wang SS, Devesa SS, Hartge P, Weisenburger DD, Linet MS: Lymphoma incidence patterns by WHO subtype in the United States, 1992-2001. Blood 2006, 107:265-276.

2. Jares $P$, Campo E: Advances in the understanding of mantle cell lymphoma. Br J Haematol 2008, 142:149-165.

3. Pileri SA, Falini B: Mantle cell lymphoma. Haematologica 2009, 94:1488-1492.

4. Komatsu H, Yoshida K, Seto M, lida S, Aikawa T, Ueda R, et al: Overexpression of PRAD1 in a mantle zone lymphoma patient with a t (11;22)(q13;q11) translocation. Br J Haematol 1993, 85:427-429.

5. Quintanilla-Martinez L, Slotta-Huspenina J, Koch I, Klier M, Hsi ED, de LL, et al: Differential diagnosis of cyclin D2+ mantle cell lymphoma based on fluorescence in situ hybridization and quantitative real-time-PCR. Haematologica 2009, 94:1595-1598.

6. Salaverria I, Espinet B, Carrio A, Costa D, Astier L, Slotta-Huspenina J, et al: Multiple recurrent chromosomal breakpoints in mantle cell lymphoma revealed by a combination of molecular cytogenetic techniques. Genes Chromosomes Cancer 2008, 47:1086-1097.

7. Mayr C, Speicher MR, Kofler DM, Buhmann R, Strehl J, Busch R, et al: Chromosomal translocations are associated with poor prognosis in chronic lymphocytic leukemia. Blood 2006, 107:742-751.

8. Decker T, Schneller F, Kronschnabl M, Dechow T, Lipford GB, Wagner H, et al: Immunostimulatory CpG-oligonucleotides induce functional high affinity IL-2 receptors on B-CLL cells: costimulation with IL-2 results in a highly immunogenic phenotype. Exp Hematol 2000, 28:558-568.

9. Haferlach C, Dicker F, Schnittger S, Kern W, Haferlach T: Comprehensive genetic characterization of CLL: a study on 506 cases analysed with chromosome banding analysis, interphase FISH, $\operatorname{lgV}(\mathrm{H})$ status and immunophenotyping. Leukemia 2007, 21:2442-2451.

10. Wren C, Moriarty H, Marsden K, Tegg E: Cytogenetic investigations of chronic lymphocytic leukemia. Cancer Genet Cytogenet 2010, 198:155-161.
11. Bacher U, Haferlach T, Schnittger S, Weiss T, Burkhard O, Bechtel B, et al: Detection of a $t(4 ; 14)(p 16 ; q 32)$ in two cases of lymphoma showing both the immunophenotype of chronic lymphocytic leukemia. Cancer Genet Cytogenet 2010, 200:170-174.

12. Karakosta M, Tsakiridou A, Korantzis I, Manola KN: Deletion of $5 q$ as a rare abnormality in chronic lymphocytic leukemia. Cancer Genet Cytogenet 2010, 200:175-179.

13. Ho AK, Hill S, Preobrazhensky SN, Miller ME, Chen Z, Bahler DW: Small Bcell neoplasms with typical mantle cell lymphoma immunophenotypes often include chronic lymphocytic leukemias. Am J Clin Pathol 2009, 131:27-32.

14. Costa ES, Pedreira CE, Barrena S, Lecrevisse Q, Flores J, Quijano S, et al: Automated pattern-guided principal component analysis vs expertbased immunophenotypic classification of B-cell chronic lymphoproliferative disorders: a step forward in the standardization of clinical immunophenotyping. Leukemia 2010, 24:1927-1933.

15. Schaffer LG, Slovak ML, Campbell L, editors: ISCN 2009: an international system for human cytogenetic nomenclature Basel: Karger; 2009.

16. Drexler HG, MacLeod RA: Malignant hematopoietic cell lines: in vitro models for the study of mantle cell lymphoma. Leuk Res 2002, 26:781-787.

17. Erdfelder F, Hertweck M, Filipovich A, Uhrmacher S, Kreuzer K-A: High lymphoid enhancer-binding factor-1 expression is associated with disease progression and poor prognosis in chronic lymphocytic leukemia. Hematology Reports 2010, 24-27.

18. Wlodarska I, Meeus $P$, Stul M, Thienpont L, Wouters E, Marcelis L, et al: Variant $\mathrm{t}(2 ; 11)(\mathrm{p} 11 ; \mathrm{q} 13)$ associated with the IgK-CCND1 rearrangement is a recurrent translocation in leukemic small-cell B-non-Hodgkin lymphoma. Leukemia 2004, 18:1705-1710.

19. Adachi M, Tsujimoto $Y$ : Juxtaposition of human bcl-2 and immunoglobulin lambda light chain gene in chronic lymphocytic leukemia is the result of a reciprocal chromosome translocation between chromosome 18 and 22. Oncogene 1989, 4:1073-1075.

20. Martin-Subero Jl, Klapper W, Sotnikova A, Callet-Bauchu E, Harder L, Bastard $C$, et al: Chromosomal breakpoints affecting immunoglobulin loci are recurrent in Hodgkin and Reed-Sternberg cells of classical Hodgkin lymphoma. Cancer Res 2006, 66:10332-10338.

21. Komatsu H, lida S, Yamamoto K, Mikuni C, Nitta M, Takahashi T, et al: A variant chromosome translocation at 11q13 identifying PRAD1/cyclin D1 as the BCL-1 gene. Blood 1994, 84:1226-1231.

22. Swerdlow SH, Campo E, Harris NL, Jaffe ES, Pileri SA, Stein H, et al: WHO classification of tumours of haematopoietic and lymphoid tissues Lyon: IARC; 2008.

23. Demiroglu A, Steer EJ, Heath C, Taylor K, Bentley M, Allen SL, et al: The t $(8 ; 22)$ in chronic myeloid leukemia fuses BCR to FGFR1: transforming activity and specific inhibition of FGFR1 fusion proteins. Blood 2001, 98:3778-3783.

24. Baxter EJ, Hochhaus A, Bolufer P, Reiter A, Fernandez JM, Senent L, et al: The $t(4 ; 22)(q 12 ; q 11)$ in atypical chronic myeloid leukaemia fuses $B C R$ to PDGFRA. Hum Mol Genet 2002, 11:1391-1397.

25. Martin-Subero Jl, Harder L, Gesk S, Schlegelberger B, Grote W, MartinezCliment JA, et al: Interphase FISH assays for the detection of translocations with breakpoints in immunoglobulin light chain loci. Int J Cancer 2002, 98:470-474.

26. Espinet B, Salaverria I, Bea S, Ruiz-Xiville N, Balague O, Salido M, et al: Incidence and prognostic impact of secondary cytogenetic aberrations in a series of 145 patients with mantle cell lymphoma. Genes Chromosomes Cancer 2010, 49:439-451.

27. Shimizu N: Extrachromosomal double minutes and chromosomal homogeneously staining regions as probes for chromosome research. Cytogenet Genome Res 2009, 124:312-326.

28. Stefancikova L, Moulis M, Fabian P, Falkova I, Vasova I, Kren L, et al: Complex analysis of cyclin D1 expression in mantle cell lymphoma: two cyclin D1-negative cases detected. J Clin Pathol 2009, 62:948-950.

29. Fernandez V, Salamero O, Espinet B, Sole F, Royo C, Navarro A, et al: Genomic and gene expression profiling defines indolent forms of mantle cell lymphoma. Cancer Res 2010, 70:1408-1418.

doi:10.1186/1755-8166-4-8

Cite this article as: Krings Rocha et al.: A rare case of $t(11 ; 22)$ in a mantle cell lymphoma like B-cell neoplasia resulting in a fusion of IGL and CCND1: case report. Molecular Cytogenetics 2011 4:8. 